\title{
Study on Deformation of the Two Rotors of Contra-Rotating Fan under Variable Speeds
}

\author{
Dawei Meng ${ }^{a}$, Xiaobo Sun ${ }^{\mathrm{b}}$ and Zeqi Zhang ${ }^{\mathrm{c}}$ \\ College of Electrical \& Electronic Engineering, Harbin University of Science and Technology, Harbin \\ 150040, China. \\ amengdawei@hrbust.edu.cn, bsunxiaobo1972@hrbust.edu.cn, ${ }^{c} 58348988 @ q q . c o m$
}

Keywords: contra-rotating fan, air pressure, deformation of blades.

\begin{abstract}
With the purpose of analyzing the pressure and deformation of the two rotors' blades of contra-rotating fan under variable speeds, the research takes the FBD5.0/2 $\times 7.5$ mine contra-rotating fan as an example, establishes a 3D physical model of contra-rotating fan's entire flow passage, and adopts 3D infrared scanner to have a point cloud scan on the two rotors' blades in order to form a more concrete model. Based on the 3D physical model, the boundary conditions and assumed conditions for solving the air pressure on the blade surface are given, and the air pressure of the rotors' blades is calculated in condition of four various speed ratios based on the theory of Computational Fluid Dynamics. In addition, the deformation of the two rotors' blades is calculated and analyzed. According to the calculating results above, the correlation between deformation and rotating speed, and the maximum deformation are assured. The results provide necessary theoretical base for the safe running of contra-rotating fan.
\end{abstract}

\section{Introduction}

Axial flow fan is a kind of machinery which is widely used in coal mines, tunnels and chemical projects. The contra-rotating fan is a special case, with two contra-rotating rotors, which are directly connected with the motor shaft end. Compared with ordinary axial flow fan, the contra-rotating fan has the characteristics of compact structure, low noise, high pressure, high flow rate and high efficiency [1]. When the flow rate of the fan decreases, its air pressure increases. Meanwhile the rear motor load quickly raises which is soon more than the front motor load and finally overload [2]. The operation mode that the speeds of the rotors are not equal can improve the efficiency of contra-rotating fan, broaden operating region of the fan, and then can get obvious benefits [3]. Driven by pole-changing motor, the two rotors can rotate at different speeds, and can be combined into a variety of different operating conditions, which can avoid the rear motor overloading and is suitable for different wind distance ventilation system under harsh environment [4, 5 and 6]. But the structure of the contra-rotating fan makes the inner flow field of the fan more complicated, and the force distribution of the two rotors is different from the ordinary one. In order to ensure it safety for variable speeds operating, the air pressure distribution and the deformation of the rotors at different speeds are studied.

In this paper, the flow performance of a contra- rotating fan of FBD5.0/2 $\times 7.5$ is studied on the air pressure distribution of the surface of the two rotors by computational fluid dynamics (CFD) method. The comparison of the deformation and air pressure distribution of the two rotors' blades are performed under different speed ratios. The maximum stress position of blade is determined which is the necessary base for the design and safe operation of the contra- rotating fan.

\section{Motor pole changing control principle}

In order to meet the demands of energy saving, improving the efficiency of mining machinery, and avoiding the waste of power resources, various speed matching are adopted for two rotors according to different wind distance. The driven motors are designed as pole changing ones, which can achieve $4 / 6$ pole changing. According to different working conditions, the flow fan runs in a proper flow rate region, which can reduce losses. 
The transformation of the $4 / 6$ pole of the stator windings is carried out using the phase shift method. When the windings are connected to 6 poles, the 120 degree phase band is adopted; however the 60 degree phase is adopted when the windings are connected to 4 poles. The connection modes of variable-pole motor are shown in Fig 1.

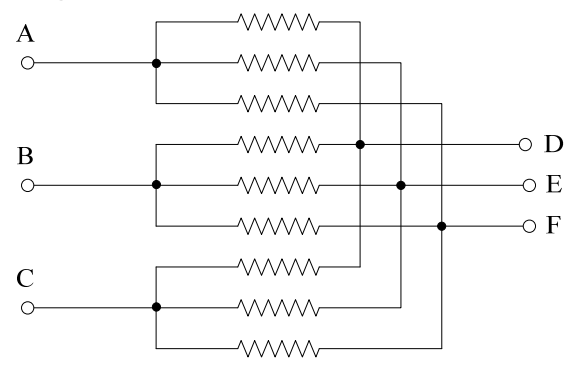

Fig.1 The connection mode of pole-change motor

For easy control, there are 6 terminals in the windings. When A, B, C are connected to the power supply, D, E, F are left vacant, the motor is 4 poles. When $\mathrm{A}, \mathrm{B}, \mathrm{C}$ are left vacant, and $\mathrm{D}, \mathrm{E}, \mathrm{F}$ are connected to the power supply, the motor is 6 poles. So the fan can rotate at different speeds to meet the requirements of different air supply distance. The air pressure and deformation of the blades under different rotational speeds are studied in the following sections.

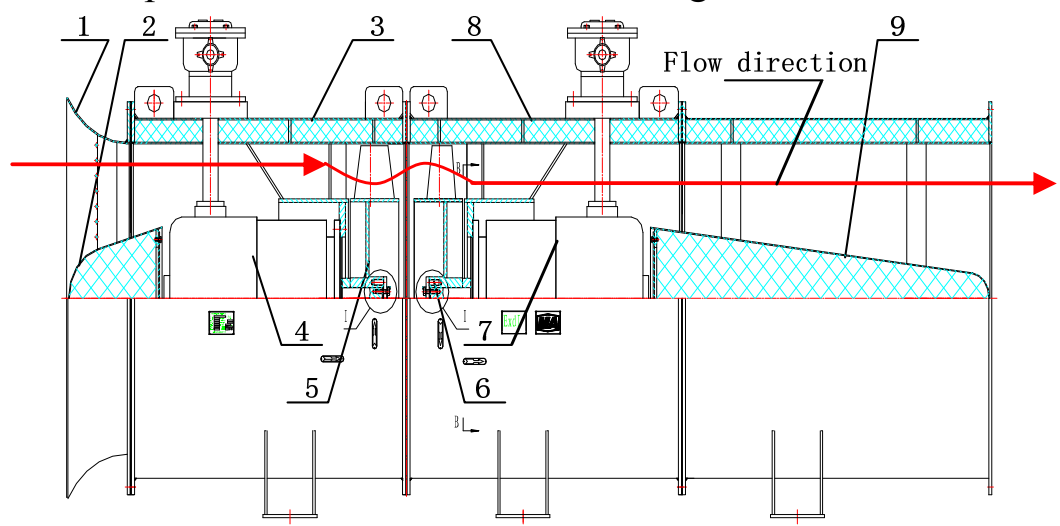

1-Air collector, 2-Nose fairing, 3-Front duct, 4-Front motor, 5-Front rotor, 6-Rear rotor, 7-Rear motor, 8-Rear duct, 9-Tail fairing

Fig.2 Structural diagram of contra-rotating fan

\section{The physical model of contra-rotating fan}

The basic structure of the contra-rotating fan. The structure of the contra rotating fan is shown in Figure 2. The two rotors are directly driven by two three phase explosion-proof induction motors. Reverse rotation of the rear rotor offsets the airflow convolution of the front rotor, so as to meet the requirements of axial flow fan outlet, which is that axial airflow velocity is larger, and tangential airflow velocity is cut to small as far as possible [7]. In this paper, the fan adopts arc plate blades, as shown in Figure 3.

Two rotors of the contra-rotating fan are used as guide vane of the other side, eliminating the guide vane of the ordinary axial flow fan, so the axial distance is small. By changing the direction of the rotation of the two rotors to achieve the backward wind transmission, in this case the flow rate can reach $60 \%$ of the forward flow rate. There is no loss of transmission loss between rotors and motors, and no loss of air flow on the guide vane, so the efficiency is improved [8]. 


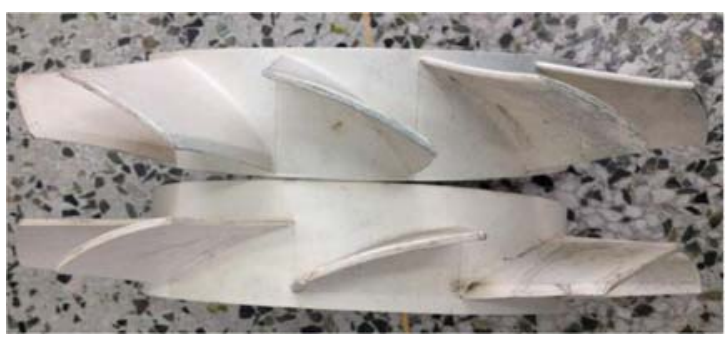

The up is front rotor, and the down is rear rotor.

Fig.3 Rotors of contrar-rotating fan

Simulation model of contra-rotating fan. The blades of FBD5.0/2 $\times 7.5$ adopt arc airfoil. The section of the blade is gradually changing from the top to the root. The number of the front rotor blades is 12 , with distortion angle $16^{\circ}$, and installation angle $43^{\circ}$. The number of the rear rotor blades is 10 , with distortion angle $4.5^{\circ}$ and installation angle $22.5^{\circ}$.

The key of modeling procedure is to convert the structure of the two rotors into a numerical model which can be identified by the simulation software. In order to accurately obtain the shape of the twisted blades, a 3-D infrared scanner is used to carry out the point cloud scanning of the two rotors blades. The results are shown in Figure 4.
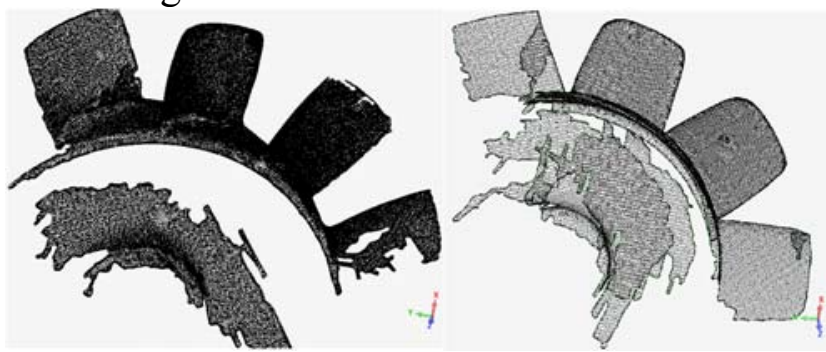

a) Front rotor point cloud b) Rear rotor point cloud

Fig. 4 Cloud image of contra-rotating fan's rotors

A combination of point cloud images after scanning ultimately generated the two rotors' model, as shown in Figure 5.

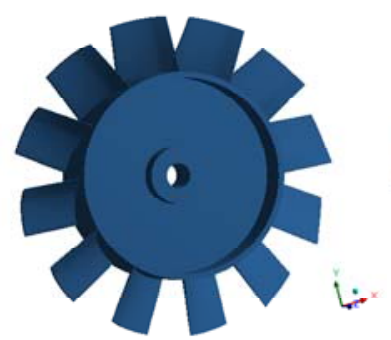

a) Front rotor

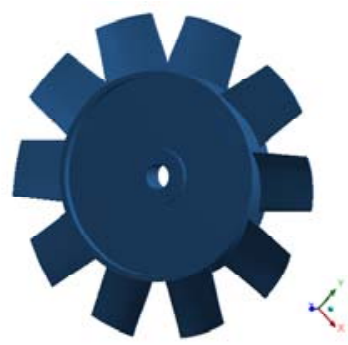

b) Rear rotor

Fig.5 The model of contra-rotating fan's rotors

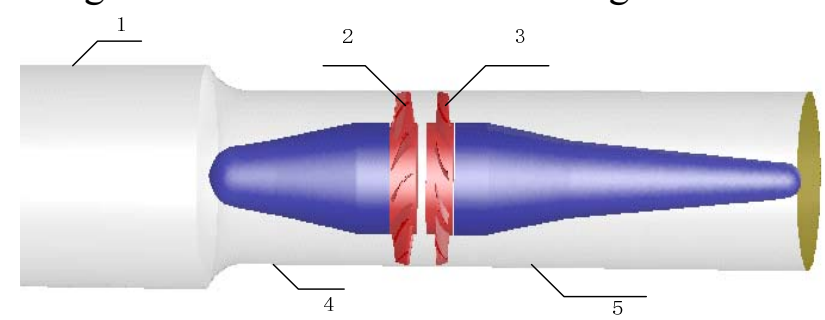

1- air collector, 2-Front rotor, 3-Rear rotor, 4-Front duct, 5- Rear duct

Fig. 6 The entire 3D numerical model of contra-rotating fan

Flow passage of the fan is composed of the collector, two ducts, two rotors and diffuser. The two rotors, rotating in the opposite direction, work on the air in smooth flow passage. The overall 3-D model of the fan is shown in Figure 6. 


\section{Analysis of the strength of the rotors}

Severe deformation of the blades and hub will affect normal production and staff safety. Mechanical strength must be checked and analyzed. The two rotors are made of nylon material (Pa66) as a whole. The material density is $1.37 \mathrm{~g} \cdot \mathrm{cm}-3$, with its elastic modulus $9100 \mathrm{MPa}$ and Poisson's ratio of 0.4 .

Blade surface pressure calculation. The rapid rotation of the rotors can make the blade surface under the action of air pressure. Air pressure and rotors rotation will have effects on the deformation of the blade. The effect of air pressure must be considered when analyzing the strength of the blade. In this paper, the CFD theory is used to analyze the air pressure of the blade surface when the fan is rotating. For simulation, the following assumptions are made.

(a)The gas is incompressible in the fan.

(b)Neglect the influence of gravity and buoyancy in the process of gas transmission in the fan.

(c) Neglect the influence of temperature.

The flow of the fan is a highly complex three-dimensional viscous one; there exists the phenomenon of vortex flow, reverse flow, laminar flow and turbulence. RNG $k-\varepsilon$ turbulence model is used in calculation. Inlet velocity flow rate boundary condition and outlet pressure boundary condition are used, with the relative standard atmospheric pressure $0 \mathrm{~Pa}[9,10]$.

Based on the assumption and the given conditions, the pressure distributions of the blade under different speed ratios 1450/1450, 1450/960, 960/1450, and 960/960 are calculated by using numerical calculation method. The windward and leeward pressure distributions of blade are shown in Figures 7 and 8 .

From Figures 7 and 8, it can be seen that, no matter what kind of speed ratios, the static pressure values on the windward side are larger than that of the leeward side, and that the higher the rotational speed, the greater the static pressure differences between two sides. From the static pressure distribution on the windward side, it can be found that the static pressure at the trailing edge of the blade is larger, that the pressure value is gradually decreased from the rear to the front, and that the static pressure near the top of the blade is small. These phenomenon indicate that the air flow near the blade tip is more serious, which should be avoided as far as possible when designing the fan blades. According to the air pressure distributions of the two rotors' blade surfaces, the deformations of the two rotors' blades in different speeds ratio are analyzed.

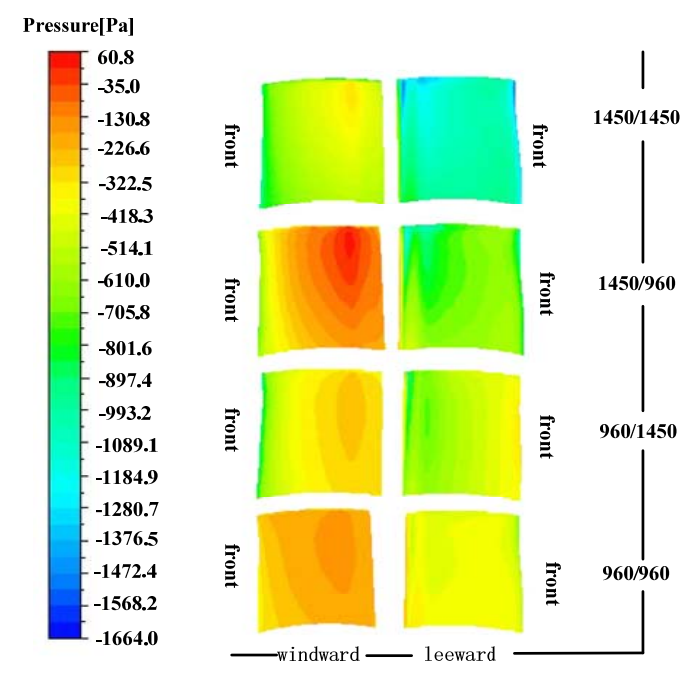

Fig.7 The pressure distributions on the windward $\&$ leeward surface of front rotor blades in different speed ratios 


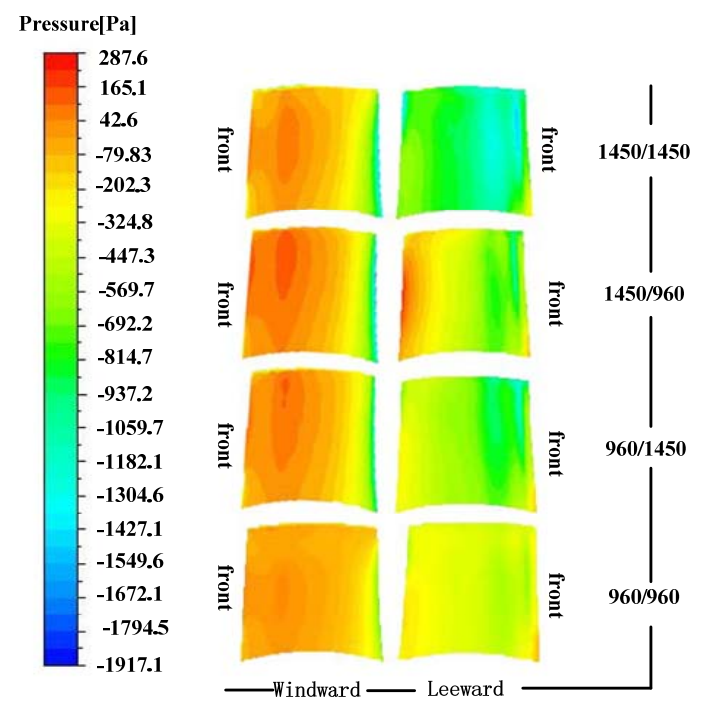

Fig. 8 The pressure distributions on the windward $\&$ leeward surface of rear rotor blades in different speed ratios

Analysis of the deformations of the rotors in different speed ratios. A transitional fit is adopted between the shaft hole of the rotors and the motors' shaft, with flange plate fixation. The rotors can rotate freely but not move along shaft axis. Therefore, two sides of the shaft hole and shaft need to implement a fixed constraint [11]. Loads on the blades of rotors are:

(a) Gravity acceleration load. The load is caused by the weight of the rotors themselves, and the gravity acceleration is taken as $\mathrm{g}=9.81 \mathrm{~m} \cdot \mathrm{s}-2$.

(b) Centrifugal force load. The load is caused by the rotation of the rotors themselves. In the numerical simulation, the centrifugal force is loaded by applying the rotating angular velocity.

(c) Air pressure load. The load is a distributed force, which is caused by the blade to the air flow. The calculation results of the air pressure on the blade surface are directly transferred into the structure of the blade by Ansys Workbench fluid solid coupling module.

Figure 9 shows the deformation distribution of the blades of the rotors in four speed ratios, 1450/1450, 1450/960, 960/1450, and 960/960.

From Figure 9, it can be found that, when the front rotor speed is same, there is no change in the deformation of the front rotor blades with difference of the rear rotor speed. When the front rotor speed is $1450 \mathrm{rpm}$, the deformation is kept within $0.01 \sim 0.05 \mathrm{~mm}$. When the front rotor speed is 960rpm, the deformation is kept within $0.005 \sim 0.020 \mathrm{~mm}$. The change of the front rotor speed causes little change of the deformation distribution of the front rotor.

It is also found that, when the rear rotor speed is same, there is little change in the deformation of the rear rotor blades with difference of the front rotor speed. When the rear rotor is 1450rpm, the deformation is kept within $0.008 \sim 0.0255 \mathrm{~mm}$. When the rear rotor speed is $960 \mathrm{rpm}$, the deformation is kept within $0.004 \sim 0.013 \mathrm{~mm}$. Also, the change of the rear rotor speed causes little change of the deformation distribution.

It can be seen from Figure 9 that, although the two rotors' speed ratio is not same, the deformation distributions of the blades are almost the same. The deformation of the front rotor blades is gradually increased from the root to the top. The maximum deformation is reached at the top of the blade, and the deformation of the front rotor blades at the blade root is the smallest. This is due to the fixation on the hub of the blade, which limits the deformation. The deformation distributions of the rear rotor blades is gradually increased from the tailing edge of the blade root to the front edge of the blade root, and the deformation at the root of the leaf is the smallest. The reason is related to the inherent structure of the blade, and the air pressure of the blade. 


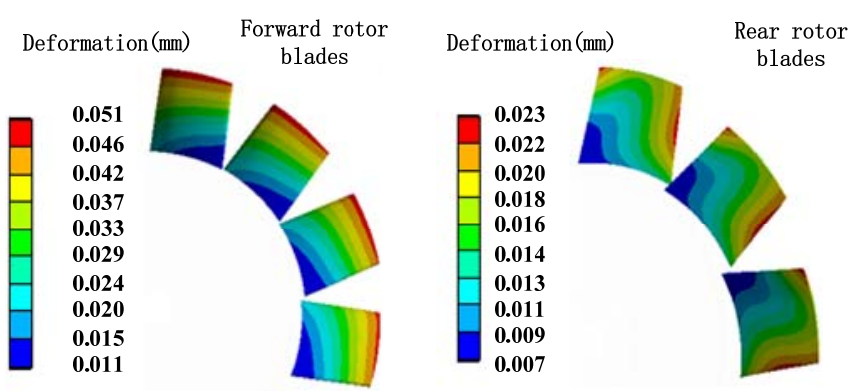

a) Two rotors' speed ratio is $1450 / 1450$

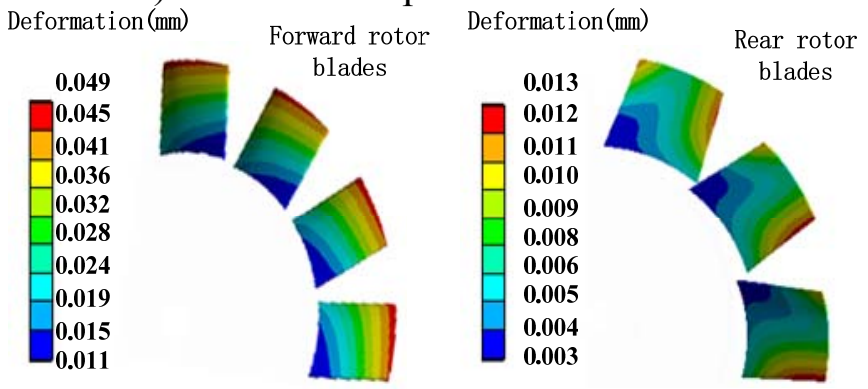

b) Two rotors' speed ratio is $1450 / 960$

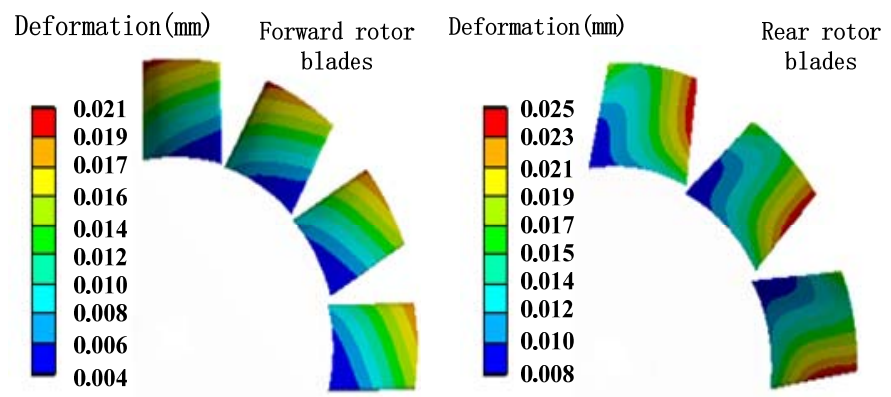

c) Two rotors' speed ratio is $960 / 1450$
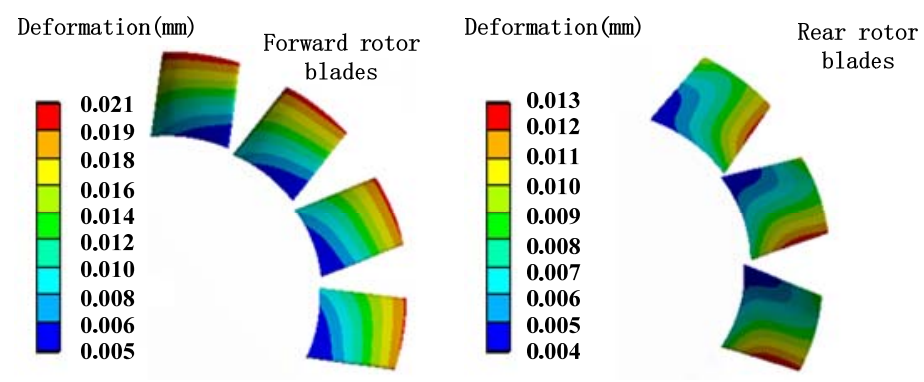

d) Two rotors' speed ratio is $960 / 960$

Fig.9 The deformation distributions of the rotors' blades in different speed ratios

Figure 10 shows that the deformation distribution of the hubs of the two rotors at different rotation speed ratios. It can be seen that the change of two rotors speed does not cause large changes in the distribution of deformation of the hub rings, and that the deformation of front hub ring increases gradually from the air inlet direction, however that of rear hub ring decreases gradually from the air inlet direction. Contours and vectors in Figure 10 show that the deformation at the center of the hub ring is largest, and the deformation direction is from inside to outside. In the position of the hub rings, the difference of air pressure does not cause the difference of the deformation of hub ring, which is only related with the rotational speed of rotors because there are no velocity and direction changes of uniform flow along the hub ring surface, and less air pressure caused by rotors work. The value of deformation at the speed of 1450rpm is about 2 times that of the deformation at the speed of 960rpm. 


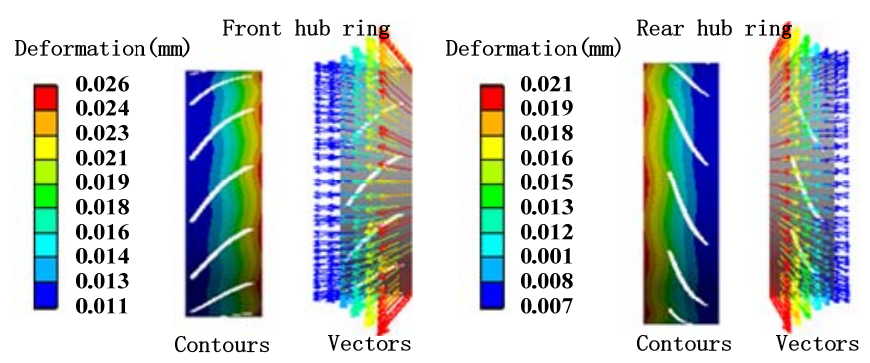

a) Two rotors' speed ratio is $1450 / 1450$

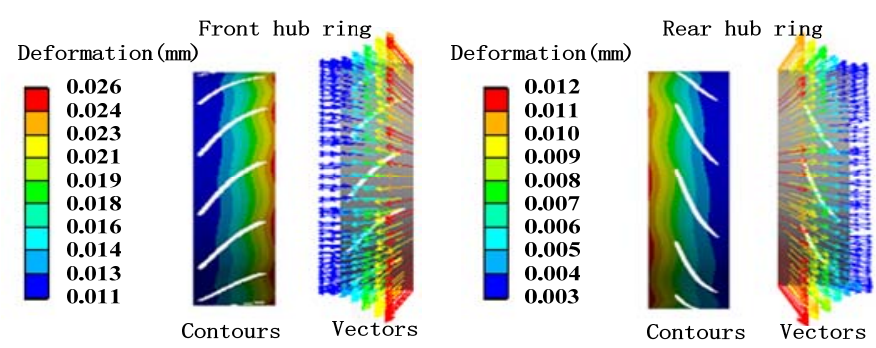

b) Two rotors' speed ratio is $1450 / 960$

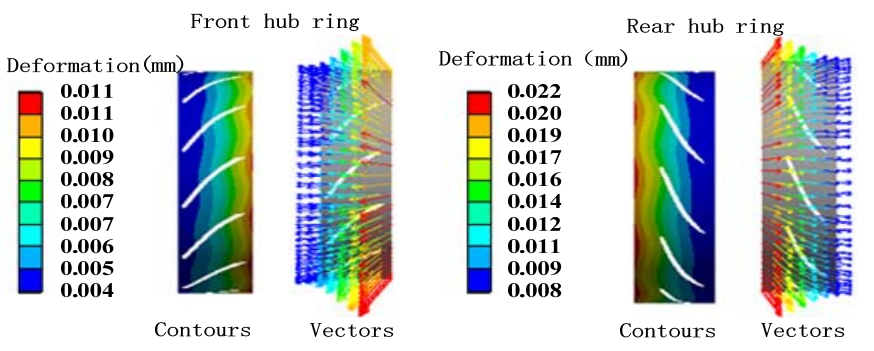

c) Two rotors' speed ratio is $960 / 1450$
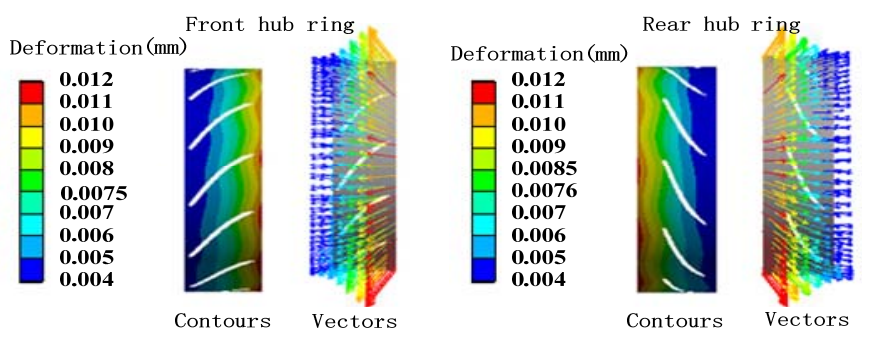

d) Two rotors' speed ratio is $960 / 960$

Fig. 10 The deformation distributions of two hub rings in different speed ratios

\section{Conclusions}

In this paper, a contra-rotating fan, FBD5.0/2 $\times 7.5$, is simulated under consideration of the effects of gravity, centrifugal force and surface air pressure on rotors. The distribution and deformation of the air pressure of two rotors under four speed ratios were studied. A variety of speed matching of contra-rotating axial flow fan was realized using motor pole changing control. Through the finite element fluid solid coupling module of Ansys workbench, the air pressure values of the blade in Fluent are accurately introduced into the structure of the blades, which makes the calculation easy.

The main conclusions arrived at from the present study are:

(a) The blade shape deformation increases with the increase of rotational speed, and the deformation distribution will not change with the rotation speed.

(b) No matter what kind of speed ratios, the static pressure values on the windward side is larger than that of the leeward side, and that the higher the rotational speed, the greater the static pressure differences between two sides.

(c) When the front/rear rotor speed is same, there is little change in the deformation of the front rotor blades under different the rear/front rotor speed. 
(d) The changes of two rotors' speeds have little effects on the distribution of deformation of the hub rings. The deformation of front hub ring increases gradually from the air inlet direction, however that of rear hub ring decreases gradually.

(e) The deformation at the center of the hub ring is largest, and its direction is from inside to outside. In the position of the hub rings, the difference of air pressure has little effects on the deformation of hub ring, which is only related with the rotational speed of rotors.

From the above conclusions, the deformation of the two rotors has little effects to the safe operation of contra-rotating fan under variable speeds. Further experiments should be done to verify the simulation results and it is proper to drive contra-rotating fan by pole-changing motors.

\section{Acknowledgements}

The authors wish to show our special thanks to the support by National Natural Science Foundation of China (No.51275137).

Corresponding author' email: sunxiaobo1972@hrbust.edu.cn

\section{References}

[1] Xie Wei, Zhang Yongjian, Huo Zuliang, Problems existed in design and solving method of counter-rotating axial flow fan for mine, Compressor, Blower \& Fan Technology, (2005) No.4, pp. 47-49.

[2] Huang Jiawei, Deng Haishun, Overload analysis and improved design for two-step motor of counter-rotating fan, Compressor Blower \& Fan Technology,(2004) No.5, pp.14-16.

[3] Li Qiushi, Lu Yajun, Yu Qing, Exploring the means of raising pressure and stable range of contra-rotating axial compressor system, Journal of Beijing University of Aeronautics and Astronautics, Vol.26,(2000) No.6, pp.669-672.

[4] Huang Liyan, Chen Hu, Design of impeller and strength checking of wheel based on axial-flow fan, Coal Mine Machinery, Vol.34,(2013) No.5, pp.20-21.

[5] Shigemitsu T, Fukutomi J, Okabe Y, Performance and flow condition of small-sized axial fan and adoption of contra-rotating rotors, Journal of Thermal Science, Vol.19, (2010) No.1, pp.1-6.

[6] Zhou Bing, Chen Naixiang, Numerical simulation of 3-D steady turbulent flow in a double channel impeller, Journal of Tsinghua University (Science and Technology), Vol.45, (2005) No.8, pp.1130-1133.

[7] Lee K S, Kim K Y, Samad A., Design optimization of low-speed axial flow fan blade with three-dimensional RANS analysis, Journal of Mechanical Science and Technology, Vol.22, (2008) No.10, pp.1864-1869.

[8] Liang Xizhi, Wu Hai, Zhao Zhen, Design and experimental investigation of contra-rotating axial flow fan, Journal of China Coal Society, Vol.24, (1999) No.1, pp.94-96.

[9] Liu Yang, Yang Zhigang, Application of different numerical simulation methods in axial-flow fan, Compressor, Blower \& Fan Technology, (2014) No.1, pp.24-30.

[10] Wang Fujun, Computational fluid dynamics analysis-CFD principle and applications, Tsinghua University Press, Beijing, 2004.

[11] He Qiudong, Gong Shuguang, Ding Tao, (2011), Analyses on blade fracture of contra-rotating axial fan for mine local ventilator and its improvement measures, Journal of Mechanical Engineering, Vol.47, (2011) No.24, pp.122-127. 